\title{
Mismatch Errors in the Measurement of Ultrahigh-Frequency and Microwave Variable Attenuators
}

\author{
R. W. Beatty
}

\begin{abstract}
The mismatch error in the measurement of ultrahigh-frequency and microwave variable attenuators is analyzed and expressions are derived. An example is given to show that the mismatch error in measuring the difference in attenuation between two attenuators is less than the sum of the mismatch errors obtained when measuring each attenuator individually.
\end{abstract}

\section{Introduction}

The error resulting from mismatched generator and detector sections in the measurement of the attenuation of a single attenuator is well known. ${ }^{1}$ The corresponding error in the measurement of changes in attenuation is important in the calibration of variable attenuators and in the calibration of large attenuators, using known pads as gage blocks or fixed attenuation standards.

The calibration of a variable attenuator consists in measuring the change in the insertion loss as the attenuator dial moves from a zero or reference position to another position that is marked or can be read on a scale. The change in the insertion loss equals the change in attenuation if the attenuator is placed in a reflectionless, or matched, system. There is always a degree of uncertainty regarding the match, depending upon the accuracy of the instruments used to indicate or recognize matched conditions and upon the reflections from connectors. For this reason the change in the insertion loss cannot be considered to be exactly equal to the change in attenuation, and the difference is called the mismatch error.

\section{Expression for Mismatch Error}

A change in a variable attenuator from the reference position to another position is equivalent to removing one attenuator and inserting another attenuator in the circuit. The insertion loss, in decibels, of the attenuator ${ }^{2}$ corresponding to the reference position of the variable attenuator is

$$
L=20 \log _{10}\left|\frac{\left(1-S_{11} \Gamma_{\mathrm{G}}\right)\left(1-S_{22} \Gamma_{\mathrm{L}}\right)-S_{12}^{2} \Gamma_{\mathrm{G}} \Gamma_{\mathrm{L}}}{S_{12}\left(1-\Gamma_{\mathrm{G}} \Gamma_{\mathrm{L}}\right)}\right|
$$

The voltage-reflection coefficieits $\Gamma_{G}$ and $\Gamma_{L}$ refer to the generator and load, respectively, and are measured at the terminals where the attenuator is inserted. The scattering coefficients ${ }^{3} S_{11}, S_{12}$, and

1 C. G. Montgomery, Technique of microwave measurements, p. 824 (McGrawHill Book Co., Inc., New York, N. Y., 1947).

2 See Appendix.

3 The scattering coefficients $S_{11}, S_{12}$, and $S_{22}$ are in effect defined by eq 12 and figure 2. See also, C. G. Montgomery, R. H. Dicke, and E. M. Purcell, Prin figure 2. See also, C. G. Montgomery, R. H. Dicke, and E. M. Purcell, Prin
ciples of microwave eircuits, p. 146-151 (McGraw-Hill Book Co., Inc., New ciples of microwave
York, N. Y., 1948).
$S_{22}$ refer to the attenuator, corresponding to the reference or zero position of the variable attenuator. The corresponding insertion loss for a different setting of the variable attenuator is

$L^{\prime}=20 \log _{10} \mid \frac{\left(1-S_{11}^{\prime} \Gamma_{\mathrm{G}}\right)\left(1-S_{22}^{\prime} \Gamma_{\mathrm{L}}\right)-\left(S_{12}^{\prime}\right)^{2} \Gamma_{\mathrm{G}} \Gamma_{\mathrm{L}}}{S_{12}^{\prime}\left(1-\Gamma_{\mathrm{G}} \Gamma_{\mathrm{L}}\right)}$.

The change in the insertion loss is

$$
\begin{aligned}
\Delta L & =L^{\prime}-L=20 \log _{10}\left|\frac{1}{S_{12}^{\prime}}\right|-20 \log _{10}\left|\frac{1}{S_{12}}\right| \\
& +20 \log _{10}\left|\frac{\left(1-S_{11}^{\prime} \Gamma_{\mathrm{G}}\right)\left(1-S_{22}^{\prime} \Gamma_{\mathrm{L}}\right)-\left(S_{12}^{\prime}\right)^{2} \Gamma_{\mathrm{G}} \Gamma_{\mathrm{L}}}{\left(1-S_{11} \Gamma_{\mathrm{G}}\right)\left(1-S_{22} \Gamma_{\mathrm{L}}\right)-S_{12}^{2} \Gamma_{\mathrm{G}} \Gamma_{\mathrm{L}}}\right|
\end{aligned}
$$

or

$$
\Delta L=A^{\prime}-A+\epsilon,
$$

where $A^{\prime}, A$, and $\epsilon$ in eq (4) correspond to the three terms in eq (3). The error $\epsilon$ must be subtracted from the change in the insertion loss to obtain the change in attenuation, $A^{\prime}-A$.

The error term can also be written ${ }^{4}$

$$
\boldsymbol{\epsilon}=20 \log _{10} \mid \frac{\left(1-\Gamma_{1}^{\prime} \Gamma_{\mathrm{G}}\right)\left(1-S_{22}^{\prime} \Gamma_{\mathrm{L}}\right)}{\left(1-\Gamma_{1} \Gamma_{\mathrm{G}}\right)\left(1-S_{22} \Gamma_{\mathrm{L}}\right)},
$$

where $\Gamma_{1}$ and $\Gamma_{1}^{\prime}$ are the input-voltage reflection coefficients of the attenuator terminated in a load having a voltage reflection coefficient $\Gamma_{\mathrm{L}}$.

The mismatch error in the measurement of a single attenuator can be obtained as a special case of eq (5). The reference attenuator vanishes in this case, changing $\Gamma_{1}$ to $\Gamma_{\mathrm{L}}, S_{12}$ to unity, and $S_{11}$ and $S_{22}$ to zero. Substituting these values for $\Gamma_{1}$ and $S_{22}$ into eq (5) yields

$$
\epsilon=20 \log _{10}\left|\frac{\left(1-\Gamma_{1}^{\prime} \Gamma_{\mathrm{G}}\right)\left(1-S_{22}^{\prime} \Gamma_{\mathrm{L}}\right)}{1-\Gamma_{\mathrm{G}} \Gamma_{\mathrm{L}}}\right|,
$$

which corresponds to eq (24), page 826 of the reference of footnote 1.

${ }^{4}$ Substitute $\Gamma_{1}=S_{11}+\frac{S_{12}^{2} \Gamma_{L}}{1-S_{22} \Gamma_{L}}$ and $\Gamma_{1}^{\prime}=S_{11}^{\prime}+\frac{\left(S_{12}^{\prime}\right)^{2} \Gamma_{L}}{1-S_{22}^{\prime} \Gamma_{L}}$ in eq (3). 


\section{Evaluation of the Mismatch Errors}

It is possible in principle to evaluate the mismatch error by measuring the voltage reflection coefficients $\Gamma_{\mathrm{G}}, \Gamma_{\mathrm{L}}, \Gamma_{1}, \Gamma_{1}^{\prime}, S_{22}$, and $S_{22}^{\prime}$, and substituting them into eq (5).

In many cases, the magnitudes of the reflection coefficients can be determined, but their phases cannot be conveniently determined because of the limitations of a particular measuring apparatus. Equation (5) can then be used to find the limits of the mismatch error, permitting the phases of the reflection coefficients to have all possible values. The limit of error can be expressed in the form

$$
\epsilon_{11 \mathrm{mit}}=20 \log _{10} \frac{\left(1 \pm\left|\Gamma_{1}^{\prime} \Gamma_{\mathrm{G}}\right|\right)\left(1 \pm\left|S_{22}^{\prime} \Gamma_{\mathrm{L}}\right|\right)}{\left(1 \mp\left|\Gamma_{1} \Gamma_{\mathrm{G}}\right|\right)\left(1 \mp\left|S_{22} \Gamma_{\mathrm{L}}\right|\right)}
$$

and the corresponding limit of error for single attenuators is

$$
\epsilon_{1 \mathrm{mit}}=20 \log _{10} \frac{\left(1 \pm\left|\Gamma_{1} \Gamma_{\mathrm{G}}\right|\right)\left(1 \pm\left|S_{22} \Gamma_{\mathbf{L}}\right|\right)}{1 \mp\left|\Gamma_{\mathrm{G}} \Gamma_{\mathrm{L}}\right|} .
$$

For example, in order to reduce mismatch errors in the calibration of attenuators, the magnitudes of $\Gamma_{G}$ and $\Gamma_{L}$ are made as small as possible, and their probable amplitude is estimated from the accuracy of the apparatus used to recognize matched conditions and from the known connector characteristics. It is difficult to accurately determine the phases of these small reflection coefficients, and the mismatch error can generally be determined not exactly, but within limits.

An example will illustrate the determination of mismatch error. If the voltage standing-wave ratios $\sigma_{\mathrm{G}}, \sigma_{\mathrm{L}}, \sigma_{1}, \sigma_{1}^{\prime}, \sigma_{22}$, and $\sigma_{22}^{\prime}[\sigma=(1+|\Gamma|) /(1-|\Gamma|)]$ corresponding to $\left|\Gamma_{\mathrm{G}}\right|,\left|\Gamma_{\mathrm{L}}\right|,\left|\Gamma_{1}\right|,\left|\Gamma_{1}^{\prime}\right|,\left|S_{22}\right|$, and $\left|S_{22}^{\prime}\right|$ are $1.1,1.1,1.2,1.5,1.2$, and 1.5 , the limits of error for the initial attenuator calculated from eq (8) are approximately \pm 0.095 decibel. The corresponding limits of error for the final attenuator are approximately \pm 0.185 decibel. The limits of error for the change in attenuation calculated from eq (7) are approximately \pm 0.242 decibel. It is seen that the mismatch error for the change in attenuation is less than the sum of the mismatch errors in measuring each attenuator individually.

It is often possible to determine the phases of $\Gamma_{1}, \Gamma_{1}^{\prime}, S_{22}$, and $S_{22}^{\prime}$, enabling one to calculate the mismatch error within narrower limits. The error in measuring voltage standing-wave ratios and phase angles produces an error in determining the limits of mismatch error but is in general a second-order effect that can be neglected.

\section{Appendix. Derivation of Expression for Insertion Loss}

The insertion loss of a four-terminal network is defined by the equation

$$
L=10 \log _{10} \frac{P_{\mathrm{L}}}{P_{2}}
$$

where $P_{\mathrm{L}}$ denotes the power delivered directly to a load, and $P_{2}$ denotes the power delivered to that load when the network is inserted between the load and the generator. It is assumed that the generator is stable and unaffected by changes in loading.

The insertion loss of a four-terminal network can be expressed in terms of its scattering coefficients $S_{11}, S_{12}$, and $S_{22}$, and the voltage reflection coefficients $\Gamma_{\mathrm{G}}$ and $\Gamma_{\mathrm{L}}$ of the generator and the load.

Referring to figures 1 and 2 , the insertion loss is

$$
L=10 \log _{10} \frac{P_{\mathrm{L}}}{P_{1}} \cdot \frac{P_{1}}{P_{2}}=10 \log _{10} \frac{P_{\mathrm{L}}}{P_{1}} \cdot \frac{1}{\eta},
$$

where $\eta$ is the efficiency of the network. If $A$ and $B$ denote the incident and reflected voltage waves shown in figure 2 . The efficiency is ${ }^{5}$

$$
\eta=\frac{P_{2}}{P_{1}}=\frac{E_{2} \cdot\left(-I_{2}\right)}{E_{1} \cdot I_{1}}=\frac{\left|B_{2}\right|^{2}-\left|A_{2}\right|^{2}}{\left|A_{1}\right|^{2}-\left|B_{1}\right|^{2}}=\left|\frac{B_{2}}{A_{1}}\right|^{2} \frac{1-\left|\Gamma_{\mathbf{L}}\right|}{1-\left|\Gamma_{1}\right|^{2}} .
$$

The scattering equations of the network are

$$
\left.\begin{array}{l}
B_{1}=S_{11} A_{1}+S_{12} A_{2} \\
B_{2}=S_{12} A_{1}+S_{22} A_{2}
\end{array}\right\},
$$

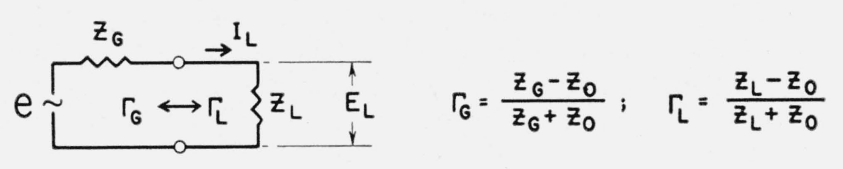

$$
\begin{aligned}
& E_{L}=e \frac{z_{L}}{Z_{G}+Z_{L}}=\frac{e}{2} \frac{\left(1-\Gamma_{G}\right)\left(1+\Gamma_{L}\right)}{\left(1-\Gamma_{G} \Gamma_{L}\right)} \\
& I_{L}=\frac{E_{L}}{z_{L}}=\frac{e}{2 Z_{0}} \frac{\left(1-\Gamma_{G}\right)\left(1-\Gamma_{L}\right)}{\left(1-\Gamma_{G} \Gamma_{L}\right)} \\
& P_{L}=E_{L} \cdot I_{L}=\frac{e^{2}}{4 Z_{0}}\left|\frac{1-\Gamma_{G}}{1-\Gamma_{G} \Gamma_{L}}\right|^{2}\left\{1-\left|\Gamma_{L}\right|^{2}\right\}
\end{aligned}
$$

Figure 1. Load connected directly to generator.

${ }^{5}$ Note that expressions of the form $E \cdot I$ represent the dot, or scalar, product of two vector quantities. 


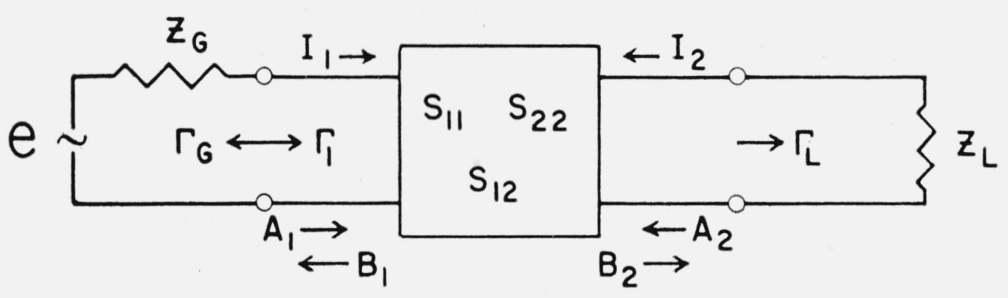

$$
\begin{aligned}
& \left\{\begin{array} { l } 
{ E _ { 1 } = A _ { 1 } + B _ { 1 } } \\
{ I _ { 1 } = \frac { 1 } { Z _ { 0 } } ( A _ { 1 } - B _ { 1 } ) }
\end{array} \quad \left\{\begin{array} { l } 
{ E _ { 2 } = A _ { 2 } + B _ { 2 } } \\
{ I _ { 2 } = \frac { 1 } { Z _ { 0 } } ( A _ { 2 } - B _ { 2 } ) }
\end{array} \quad \left\{\begin{array}{l}
\Gamma_{1}=\frac{B_{1}}{A_{1}} \\
\Gamma_{L}=\frac{A_{2}}{B_{2}}
\end{array}\right.\right.\right. \\
& P_{1}=E_{1} \cdot I_{1}=\frac{\left|A_{1}\right|^{2}-\left|B_{1}\right|^{2}}{Z_{0}}=\frac{\left|A_{1}\right|^{2}}{Z_{0}}\left\{1-\left|\Gamma_{1}\right|^{2}\right\} \\
& P_{2}=E_{2} \cdot\left(-I_{2}\right)=\frac{\left|B_{2}\right|^{2}-\left|A_{2}\right|^{2}}{Z_{0}}=\frac{\left|B_{2}\right|^{2}}{Z_{0}}\left\{1-\left|\Gamma_{L}\right|^{2}\right\}
\end{aligned}
$$

Figure 2. Network inserted between generator and load.

where $\Gamma_{1=B_{1} / A_{1}}$ and $\Gamma_{L=A_{2} / B_{2}}$. But

therefore,

$$
\frac{B_{2}}{A_{1}}=\frac{S_{12}}{1-S_{22} \Gamma_{\mathrm{L}}}
$$

$$
\eta=\left|\frac{S_{12}}{1-S_{22} \Gamma_{\mathbf{L}}}\right|^{2} \frac{1-\left|\Gamma_{\mathrm{L}}\right|^{2}}{1-\left|\Gamma_{1}\right|^{2}} \text {. }
$$

Inspection of figure 1 leads to the conclusion

$$
\frac{P_{\mathrm{L}}}{P_{1}}=\left|\frac{1-\Gamma_{\mathrm{G}} \Gamma_{1}}{1-\Gamma_{\mathrm{G}} \Gamma_{\mathrm{L}}}\right|^{2} \cdot \frac{1-\left|\Gamma_{\mathrm{L}}\right|^{2}}{1-\left|\Gamma_{1}\right|^{2}} .
$$

Substitution of these equations into eq (8) yields

$$
L=20 \log _{10} \frac{\left(1-\Gamma_{\mathrm{G}} \Gamma_{1}\right)\left(1-S_{22} \Gamma_{\mathrm{L}}\right)}{S_{12}\left(1-\Gamma_{\mathrm{G}} \Gamma_{\mathrm{L}}\right)}
$$

Since

$$
\Gamma_{1}=S_{11}+\frac{S_{12}^{2} \Gamma_{\mathrm{L}}}{1-S_{22} \Gamma_{\mathrm{L}}},
$$

the insertion loss may also be written

$$
L=20 \log _{10}\left|\frac{\left(1-S_{11} \Gamma_{\mathrm{G}}\right)\left(1-S_{22} \Gamma_{\mathrm{L}}\right)-S_{12}^{2} \Gamma_{\mathrm{G}} \Gamma_{\mathrm{L}}}{S_{12}\left(1-\Gamma_{\mathrm{G}} \Gamma_{\mathrm{L}}\right)}\right|
$$

